\title{
Remote sensing of volcanic terrains by terrestrial laser scanner: preliminary reflectance and RGB implications for studying Vesuvius crater (Italy)
}

\author{
Arianna Pesci $\left({ }^{1}\right)$, Giordano Teza $\left({ }^{2}\right)$ and Guido Ventura $\left(^{3}\right)$ \\ $\left.{ }^{(}\right)$Istituto Nazionale di Geofisica e Vulcanologia, Sezione di Bologna, Bologna, Italy \\ $\left(^{2}\right)$ Dipartimento di Geoscienze, Università degli Studi di Padova, Italy \\ $\left.{ }^{3}\right)$ Istituto Nazionale di Geofisica e Vulcanologia, Roma, Italy
}

\begin{abstract}
This article focuses on the use of terrestrial laser scanner (TLS) in the characterization of volcanic environments. A TLS survey of the Vesuvius crater (Somma-Vesuvius volcano, Italy) allows the definition of an accurate and georeferenced digital model of different sectors of the crater. In each sector, the intensity is computed for each point as the ratio between the emitted amplitude and the received one, normalized to the maximum signal. Moreover, the RGB colors of the observed surfaces can be captured by means of a calibrated camera mounted on the TLS instrument. In this way, a multi-band information is given, since a long range TLS operates in the near infrared band. The reflectance and RGB data are compared to verify their complementarities for model analysis and inspection. The collected data allowed the recognition of different volcanic deposits and stratigraphic features. In addition, our results shed light on the spatial extension of landslides and on the dimensions of rock fall/flow deposits affecting the inner walls of the crater.
\end{abstract}

Key words Terrestrial Laser Scanner - Reflectance - RGB - Volcanoes - Vesuvius.

\section{Introduction}

The terrestrial laser scanner (TLS) is generally used for the geometrical and kinematical characterization of surfaces affected by deformation allowing the definition of very accurate digital models from remotely acquired data. Hunter et al. (2003) showed an interesting TLS application for ground deformation measure-

Mailing address: Dr. Arianna Pesci, Istituto Nazionale di Geofisica e Vulcanologia, Sezione di Bologna, Via Donato Creti 12, 40128 Bologna, Italy; tel. +39 0514151416; fax.+390514151498; e-mail: pesci@bo.ingv.it ments at Mt. Etna. Teza et al. (2007) provided a method for displacement field computation of landslide ground surfaces by multi-temporal TLS-based models comparison. The new generation of very long range scanners allows surface observation operating on ranges of some hundred meters and more providing very detailed (few centimeters) digital models (Remondino, 2003). A TLS scan provides the position of acquired points and a radiometric information; for each element of the point cloud, the intensity of received pulse is measured. It is related to both the material reflectance and the relative position of instruments respect to the observed surface (Reshetyuk, 2006), and it is also highly sensitive to the water content ( $\mathrm{Lu}$ et al., 2005).

A time-of-flight terrestrial laser scanner (TLS) operates with nanosecond laser pulses 
emitted along a direction defined by two calibrated angular coordinates $(\phi$, longitude and $\alpha$, elevation). The signal is backscattered by the acquired target providing the time of flight $t$, and the path is computed as $d=c t / 2$ ( $c$ is the speed of light) leading to the complete spherical coordinates $\left(\phi_{k}, \alpha_{k}, d_{k}\right)$. The laser beam is automatically deflected over known directions by a moving mirror device providing the acquisition of a large number of points well distributed on the observed physical surface. The laser beam properties assure spatial collimation and good waveform of the pulses, leading to high resolution and accuracy. The Optech ILRIS 3D, e.g., is able to acquire a point at $50 \mathrm{~m}$ distance with about $7 \mathrm{~mm}$ accuracy and with $17.7 \mathrm{~mm}$ resolution (Lichti and Jamtsho, 2006). The accuracy is mainly related to the internal telemeter efficiency in time measuring, leading to a precise range definition, whereas the resolution depends on spot size and on the level of data overlapping, based on the choice of the calibrated angular grid.

The product of a scan is the raw point cloud $\left\{\left(d_{k}, \phi_{k}, \alpha_{k}, I\left(d_{k}, \phi_{k}, \alpha_{k}, d_{k}\right)\right), \quad k=1,2, \cdots, N\right\}$, where $I\left(d_{k}, \phi_{k}, \theta_{k}, d_{k}\right)$ is the intensity of the pulse corresponding to the $k$-th point and $N$ is the number of acquired points (generally, $N \sim 10^{5}-10^{6}$ ). The raw point cloud is directly provided by the instrument. The spherical coordinates are transformed into Cartesian ones and the intensity is normalized to the range $0-255$ in order to allow the data importing by software intended for TLS data processing.

When a calibrated camera is mounted on the TLS instrument, a series of RGB images of the observed scene can be acquired. The RGB and TLS-based data can be also merged by means of specific software leading to a colored point cloud or a textured digital model. Since a camera is a passive instrument, the RGB image critically depends on the light conditions. On the contrary, since the TLS is an active instrument, the acquisition is independent from light conditions.

Both the intensity and RGB data can be useful in point cloud inspection for edge detection and in the identification of different materials (Kurazume et al., 2002). Roncella et al. (2005) showed that a 3D model obtained from digital photogrammetric techniques can be used to recognize geo-mechanical features, since image processing operators conceived for image segmentation (e.g., edge detection operators) can be applied.

At present, few studies make specific use of the intensity data obtained by TLS or aerial laser scanner instruments (e.g. Mazzarini et al., 2007), but the geometric information is principally used.

This paper investigates the nature of TLSderived intensity and coupled RGB data to verify if: a) the corresponding information is redundant, or an integration could be useful or necessary, and b) the intensity and/or RGB data can be used to recognize different material and to detect, for example, stratigraphic successions. The crater of Vesuvius (Italy) is the test site selected to investigate the peculiarities of a volcanic environment.

\section{Terrestrial laser scanner: fundamentals and properties of acquired data}

As stated above, the TLS point cloud is $\left\{\left(x_{k}, y_{k}, z_{k}, I_{n}\left(x_{k}, y_{k}, z_{k}\right)\right), k=1,2, \cdots, N_{e}\right\}$, where $I_{n}$ is the normalized intensity and $N_{e} \cong N$ is the number of points where the intensity can be effectively considered (for example, a signal saturation in the internal sensor consists of corresponding point removal). The raw intensity $I$, directly provided by the instrument, is converted into the normalized intensity $I_{n}$ by means of: a) target-distance corrections, and $b$ ) transformation of obtained results into the 0-255 range of integers, like a grey level image. The target-distance correction is applied to provide similar intensity values for a same material acquired at different distances, taking into account the obvious fact that the raw intensity depends on the inverse square distance. In a straightforward approach, the distance-corrected intensity can be $I_{C, k}=I_{k}\left(d_{k} / d_{0}\right)^{2}$, where $d_{0}$ is a reference distance (generally unknown to the user), resulting in $I_{n, k}=\left[255\left(I_{C, k}-I_{C, \text { min }}\right) /\left(I_{C, \max }-I_{C, \min }\right)\right]$, where $[r]$ indicates the nearest integer to the real number $r$, and $I_{C, \max }, I_{C, \min }$ are the maximum and minimum values of the set of distance-corrected intensities. In this way, the point cloud 
can be inspected using TLS data processing software (e.g. Innovmetric PolyWorks ${ }^{\mathrm{TM}}$, ISiTE Studio ${ }^{\mathrm{TM}}$, and others). This transformation is performed using a parsing software provided by TLS manufacturer (e.g. Optech Parser). The actually implemented algorithm, where some elaborations are joined to the straightforward approach to improve the visualization, is deemed to be proprietary information and is therefore unknown.

The rate of the backscattered signal depends on both target physical properties and geometrical conditions, that is the relative position of TLS and observed surface, or better the angle of incidence. A common material is generally characterized by a quasi-Lambertian behavior, diffusing the incident radiation on the semispace, mainly concentrated along the preferential scattering direction, depending on the microscopic surface irregularities of the observed object (roughness), on the laser wavelength and on the incident angle (Baltsavias, 1999). Theoretically, a Lambertian reflection is a complete and total signal diffusion along each possible direction in the semi-space over the shot element.

A good practice of TLS observation requires the incidence to be as normal as possible, to have maximum precision and resolution, which also depend on both instrumental performances and observational conditions. The area illuminated by a laser beam (the footprint) results from a thin-cone/plane (beam/surface) intersection, leading to a circle (or ellipse) with a small but finite diameter, linearly increasing with the distance $d$ of the target (cone height). The probability of return detection decreases if the angle between the laser beam and the normal to observed surface increases. If this angle is too high, fewer points are acquired, and the accuracy on their coordinates is worse because of footprint deformation (spreading on the surface). If the characteristic size of the macroroughness of the observed surface is similar to the footprint diameter, the intensity does not depend on observation angle because a significant part of the area illuminated by the laser is always oriented towards the instrument, i.e. is not very far from normal incidence conditions (see Appendix C).
If two areas are observed under the same conditions, i.e. similar distance and angle respect to the surface normal, the different intensities are proportional to the corresponding reflectance and could be theoretically used to discriminate between different materials. Nevertheless, the intensity strongly depends on moisture of the material, since a TLS generally operates in IR band. So, the radiometric data cannot be generally used for classification purposes if multi-temporal point clouds are considered, that is a classification can be attempted only if homogeneous data are compared. Moreover, the parsing actions have to be carefully investigated for subsequent data interpretation based on intensity values, using raw data.

Many TLS typologies exist, characterized by different wavelength, accuracy, and achievable maximum range (Boehler et al., 2003). An online description of the available instruments and processing software can be found at POB (2008). Long range instruments are very interesting in geological/geophysical applications. In fact, they allow the data acquisition also of inaccessible areas like volcanic craters or landslides since observation distances of about one kilometer or better can be reached. A TLS survey does not require contact with the observed surface but a series of high-reflectance artificial targets can be placed on the scene in order to allow the georeferencing of the point cloud. These targets, whose centers are the ground control points (GCPs), can be concurrently acquired by TLS and topographical techniques like total station and/or GPS. This operation can be useful to provide some reference point for a next data registration on external frames. The GCP positions can be chosen on the basis of accessibility, the only requirement is their good spatial distribution. Some instruments are equipped with a calibrated camera and directly provide both intensity and RGB colors of the point cloud.

A specific processing procedure, usually performed by means of specific software, is required to extract useful information from the TLS data and to generate a final and complete digital model (Remondino, 2003). If an object is observed from more than one viewpoint (for example, Vesuvius crater), the processing starts 
with the registration of the point clouds, that is their linking on a common reference frame to provide a global point cloud. Generally, surface matching algorithms are used for the data registration, e.g. the Iterative Closest Point (ICP) algorithm (Besl and McKay, 1992; Chen and Medioni, 1992). The global point cloud is then georeferenced by using a series of GCPs. A data filtering/cleaning could be necessary, especially if elements to remove (e.g., trees) are present. Some expedients are used to better solve for the possible presence of vegetation, like the choice of the last pulse mode for data acquisition, providing the last arrival time for pulse detection and increasing the possibility to receive a terrain backscattered signal. Moreover, the intensity values can also be used to identify, select and remove the points corresponding to vegetation, due to water-content leading to low reflection.

The generation of a 3D polygonal model is the central point of data processing. It is a triangular mesh whose vertices are the points of the filtered and/or smoothed point cloud. The texture associated to the mesh can contain information on reflectance, RGB color, as well as on physical properties of the material.

\section{Study area}

The Somma-Vesuvius volcano consists of two main edifices: the Somma edifice, which is affected by a summit caldera, and the Vesuvius cone (1281 $\mathrm{m}$ a.s.l.), which is located within the caldera (fig. 1; Santacroce, 1987). The Somma products, which include lava flows and scorias, are older than $18.3 \mathrm{ky}$. The Somma caldera resulted from collapses related to four Plinian eruptions occurred between 18 ky and AD 79. Sub-Plinian eruptions also occurred between 16.1 ky and AD 1631 (Andronico et al., 1996). Lava flows in alternation with explosive phases characterized the activity between AD 79 and $\mathrm{AD}$ 472, and mainly between AD 1637 and 1944 (Arnò et al., 1987).

The present-day Vesuvius crater formed during the last AD 1944 eruption (Santacroce, 1987). The volcano is in a quiescent stage and only seismic and fumarolic activity occurs. The volcanic hazard is, however, high because of 600,000 people live around the Vesuvius and an inverse relation between the duration of the quiescent periods and energy of the eruptions exists (Santacroce, 1987).

The present-day inner walls of the Vesuvius crater, which measures about $550 \mathrm{~m}$ in diameter, consist of lavas related to the pre-1906 activity (southwestern wall) and of lavas of 19131944 activity (northeastern wall), as depicted in fig. 1. Spatters and pyroclastic deposits cover the Vesuvius cone and outcrop on the northeastern inner wall of the crater. The crater floor is filled by slide deposits resulting from the downfall of lava fragments/blocks outcropping on the steep, inner walls. The crater rim results from the superimposition of the northeastern rim related to the 1944 eruption on that of the pre1944 period of activity (southeastern rim). Following Ventura et al. (2005), the collapse of the small cone located before the 1944 eruption, as well as the sharp contact between the pre-1906 and 1913-1944 lavas of the inner crater walls is guided by a NW-SE striking fracture. The strike of this fracture is that of the main structural discontinuity affecting the whole Somma-Vesuvius edifice and crossing the Vesuvius cone (Bianco et al., 1998).

\section{Data acquisition}

The Vesuvius crater was observed twice times by TLS, on May 2005 (Pesci et al., 2007a) and October 2006 respectively. The instrument used was the Optech ILRIS 3D TLS (May 2005) and its upgraded, extended range (ER) version ILRIS 3D ${ }^{\mathrm{ER}}$ (October 2006). The ER version can operate in two modes, extending the maximum achievable range if necessary. This option is particularly useful in a volcanic environment where low-reflectance lavic material exists.

The whole crater was observed from different viewpoints located in the crown, chosen in order to operate with good incident angles as normal as possible, providing the total surface coverage (fig. 2). In the 2005 measurement campaign the access to the northern part of the crater was forbidden, while in October 2006 

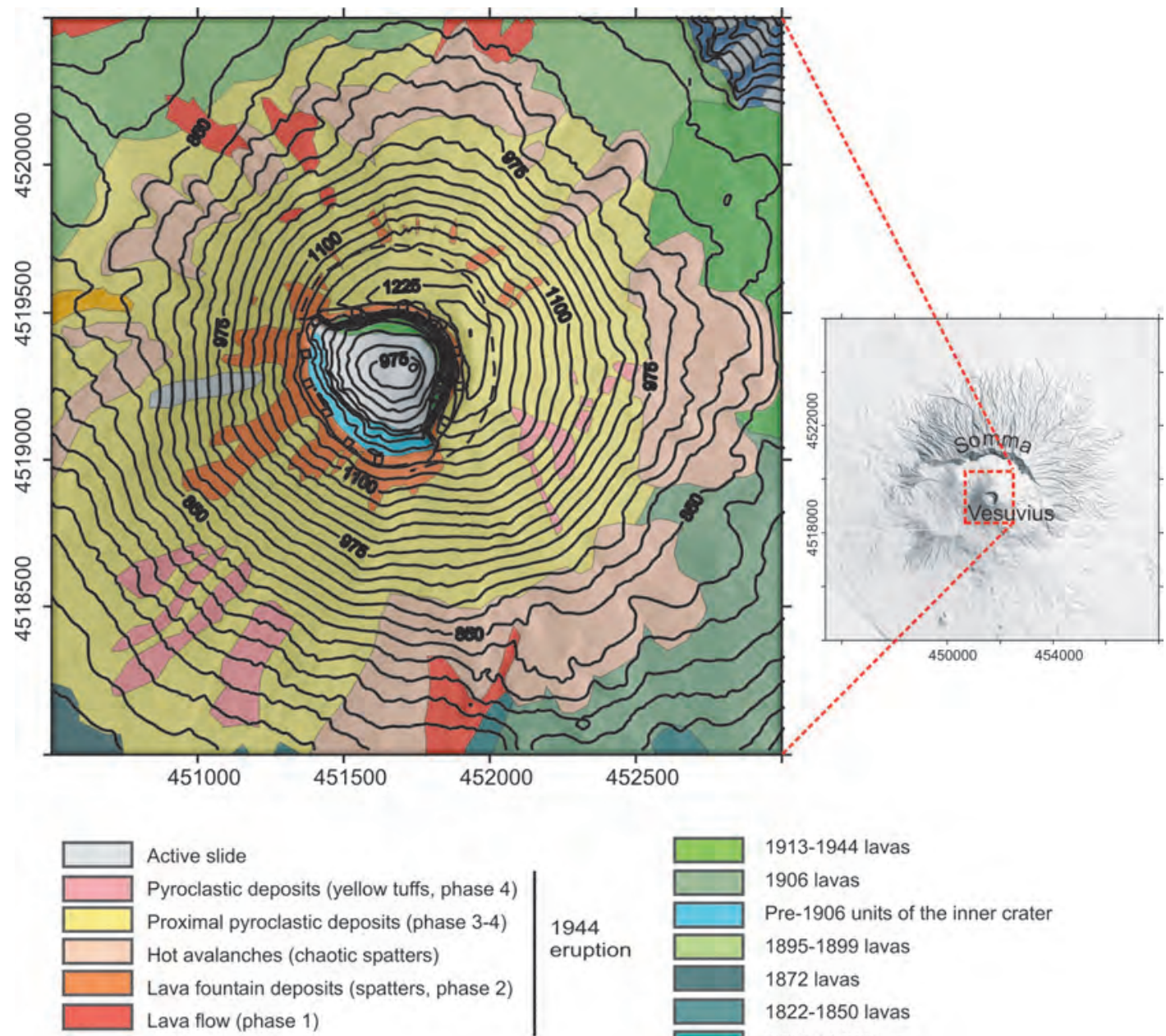

Active slide

Pyroclastic deposits (yellow tuffs, phase 4)

Proximal pyroclastic deposits (phase 3-4)

Hot avalanches (chaotic spatters)

1944

Lava fountain deposits (spatters, phase 2)

eruption

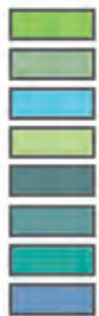

1913-1944 lavas

1906 lavas

Pre-1906 units of the inner crater

1895-1899 lavas

1872 lavas

Lava flow (phase 1)

1822-1850 lavas

1737-1847 lavas

Caldera and Pre-caldera deposits

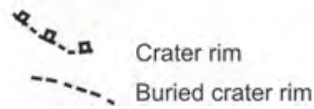

Fig. 1. Digital terrain model of the Somma-Vesuvius volcano and simplified geological sketch map of the Vesuvius crater (modified from Santacroce, 1987). The reference frame is the WGS84 (UTM coordinates).

five station points well distributed around the crater were used.

Each single scan covered the angular space of about $40^{\circ}$, and the acquisition distance ranged from $300 \mathrm{~m}$ to $600 \mathrm{~m}$. At $500 \mathrm{~m}$ distance the mean sampling step at the ground was about $5 \mathrm{~cm}$. For the used instrument, the footprint di- ameter on a surface orthogonal to the laser beam is $D=12+0.17 d$, where $d$ is the distance expressed in $\mathrm{m}$ and $\mathrm{D}$ is in $\mathrm{mm}$. Therefore, in this application $D$ ranged from $6 \mathrm{~cm}$ to $11 \mathrm{~cm}$. Since the resolution depends on both the footprint size and the sampling step, and the first dominates because its diameter is considerably greater re- 
spect to the sampling step (Lichti and Jamtsho, 2006), $10 \mathrm{~cm}$ can be considered a good estimate of the mean actual spatial resolution.

A calibrated camera was mounted over the laser unit to provide RGB colors for texturing; the camera was characterized by $28 \mathrm{~mm}$ focal distance and $0.007 \mathrm{~mm}$ pixel size, so the corresponding pixel size on the ground surface was about $12.5 \mathrm{~cm}$ at $500 \mathrm{~m}$ distance. The ground spatial resolution of camera and TLS were about the same in similar observational conditions. In this way, a comparison between intensity and RGB data, as well as an integration of these data to provide a better knowledge of the observed structures, were allowed.

The standard data processing is performed using the Innovmetric PolyWorks ${ }^{\mathrm{TM}}$ software package, providing a georeferenced 3D polygonal model for each survey. These models were also integrated with a digital terrain model (DTM) of the volcano generated by using data obtained from aerial photogrammetry enlarging the surveyed area (see fig. 2). Moreover, a preliminary comparison between 2005 and 2006 models was presented and discussed, pointing out the capability of this surveying methodology to recognize and measure surface variations in a volcanic environment (Pesci et al., 2007b).

The present article presents the main results of an analysis aimed to understand the importance of radiometric contributions to the point cloud and image inspection in a volcanic environment. In particular, the analysis is focused on three selected zones of the crater characterized by different origin, morphology and visible features (fig. 2). A-zone is the fracture area that coincides with the morphological discontinuity separating the two half parts of the crater and differ for age and lithology and was observed from about $500 \mathrm{~m}$ distance. B-zone is a volcanic wall acquired in a very detailed way thanks to short range of about $50-80 \mathrm{~m}$. The last, C-zone, is a portion of a landslide lying in the NW sector of the crater, scanned from about $500 \mathrm{~m}$.

Since in each considered zone the acquisition distances were roughly constants (A and $\mathrm{C}$ : $500 \mathrm{~m}, \mathrm{~B}: \sim 60 \mathrm{~m}$ ), the observational conditions allowed a quantitative analysis of the intensity provided by TLS.

\subsection{Crater fracture zone}

The fracture area occurring at the southeastern tip of the Vesuvius crater (A-zone in fig. 2) marks the contact between the more recent and steepest north-eastern wall and the older south-eastern wall. A vertical displacement of about $1.5 \mathrm{~m}$ can be recognized in the A-zone by means of the point cloud intensity analysis. The vertical step is clearly visible in the intensity image (fig. 3b) but cannot be directly detected in the ordinary red-saturated RGB image (see fig. 3a), also due to shadows that hide morphological features. Nevertheless, some hide features in the RGB image could be detected by means of a re-balancing of the R, G and B components to create a false color image; this process, anyway, is not easy and requires manual editing and several trials.

The properties of an RGB image strictly depends on illumination conditions, in particular a shadow can hide interesting features. On the contrary, the TLS-based intensity data are obtained by active independent illumination but its relative nature, depending on geometry of observation and weather conditions, must be taken in account. On the other hand, materials characterized by the same color tones may have different physical properties and chemical composition, whereas difference in composition could be easily detected using IR signal. In conclusion, if intensity data are used, in some cases a direct and easy recognition of some features can be performed, without additional image processing efforts.

\subsection{Crater inner wall}

The B-zone (fig. 2) is a relatively small, NW-SE striking sub-vertical cliff located near the previously described fracture area. Different lithologies, which include welded scorias, massive and scoriaceous lavas, can be recognized. A first approach to remotely map volcanic rocks is the analysis of a reduced number of data extracted from some selected sections. Six vertical profiles are considered, and both intensity and RGB data are extracted to compare data distributions and searching for data correla- 

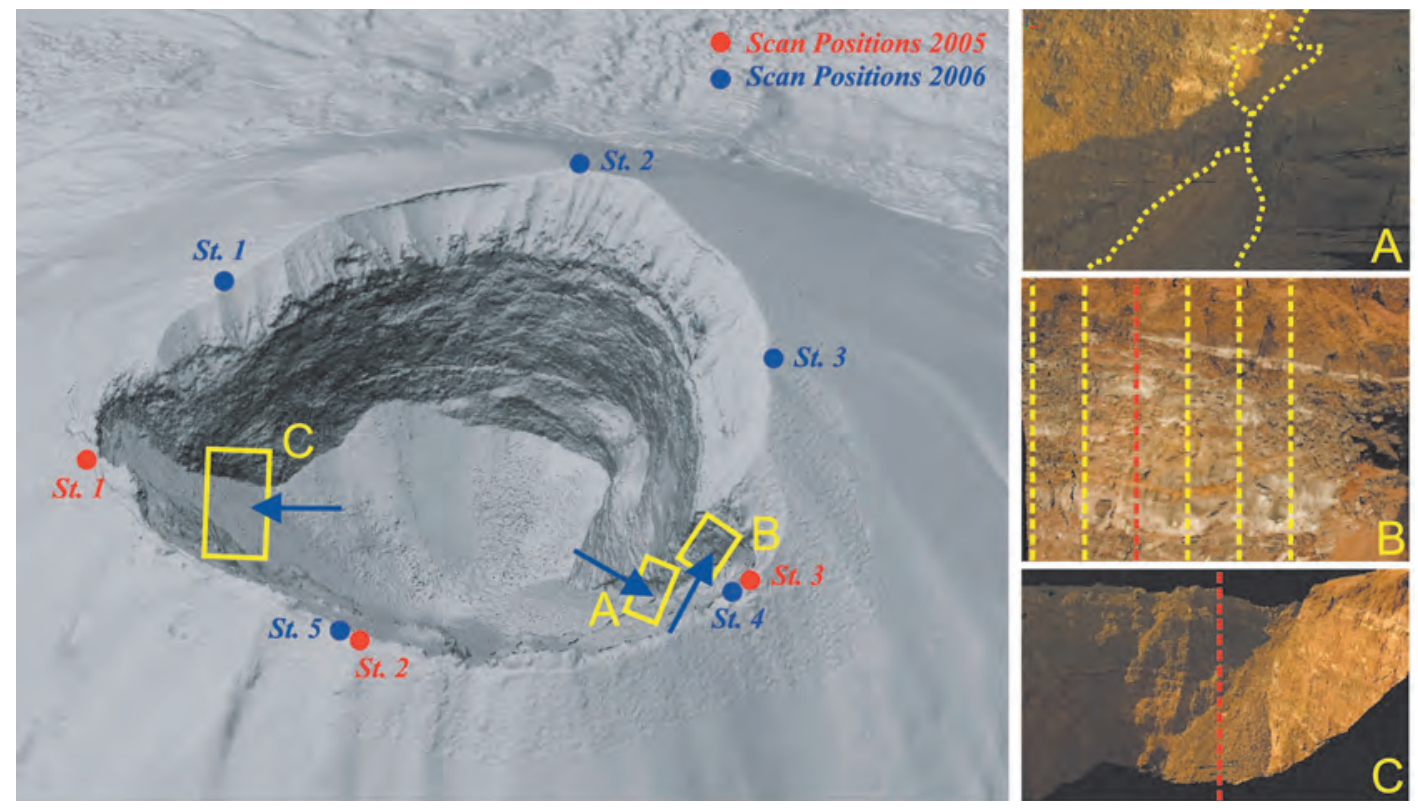

Fig. 2. Left panel: perspective view of the Vesuvius crater. The TLS viewpoints are marked by red and blue dots, concerning 2005 and 2006 surveys respectively. Lines-of-sight are reported as arrows. Right panel: front view of the three studied outcrops (yellow boxes A, B, and C in the left panel) and vertical sections used for preliminary data analysis (see later on).

tion. In particular, fig. 4 a shows the intensity statistical distribution of data extracted along the third vertical profile (from the left, the red one in fig. 2). This distribution clearly indicates preferential intensity values corresponding to histogram peaks. On the contrary, the three color channels are distributed in an almost Gaussian way leading to standard deviation values of 55, 68 and 86 for the red, green and blue component respectively. Another important result is the high correlation between the color channels, which is $0.984,0.933$ and 0.967 for the R-G, R$\mathrm{B}$ and G-B combinations respectively. Therefore, the relations among the three basic colors are here linear trends, and each channel carries nearly all the information of the RGB image. For this reason, the analyses presented there are based, besides on TLS-based IR intensity data, on the R channel only. The complete analysis is reported in Pesci et al. (2007c).

In the intensity vs. color plot of the consid- ered vertical profile, the point distribution shows a «comb-peak» shape. Despite large data dispersion, this distribution highlights the different behavior of intensity, which is organized in some defined bands, and color channels, which are spread. The same results are obtained over the other profiles (not shown here for the sake of brevity).

Dealing with a clear surface, composed of different materials distributed into irregular geometrical zones, these bands are probably related to the physical surface properties (roughness, moisture, etc.). The comb-peak structure consists of about 9-10 bands in the intensity distribution. Moreover, this structure is weakened if the entire surface is considered instead of vertical sections, due to a more noisy data set. In addition, a large number of narrow bands (with steps of some intensity units) can be observed in both the sections and the whole area. This second effect is related to the quantization 

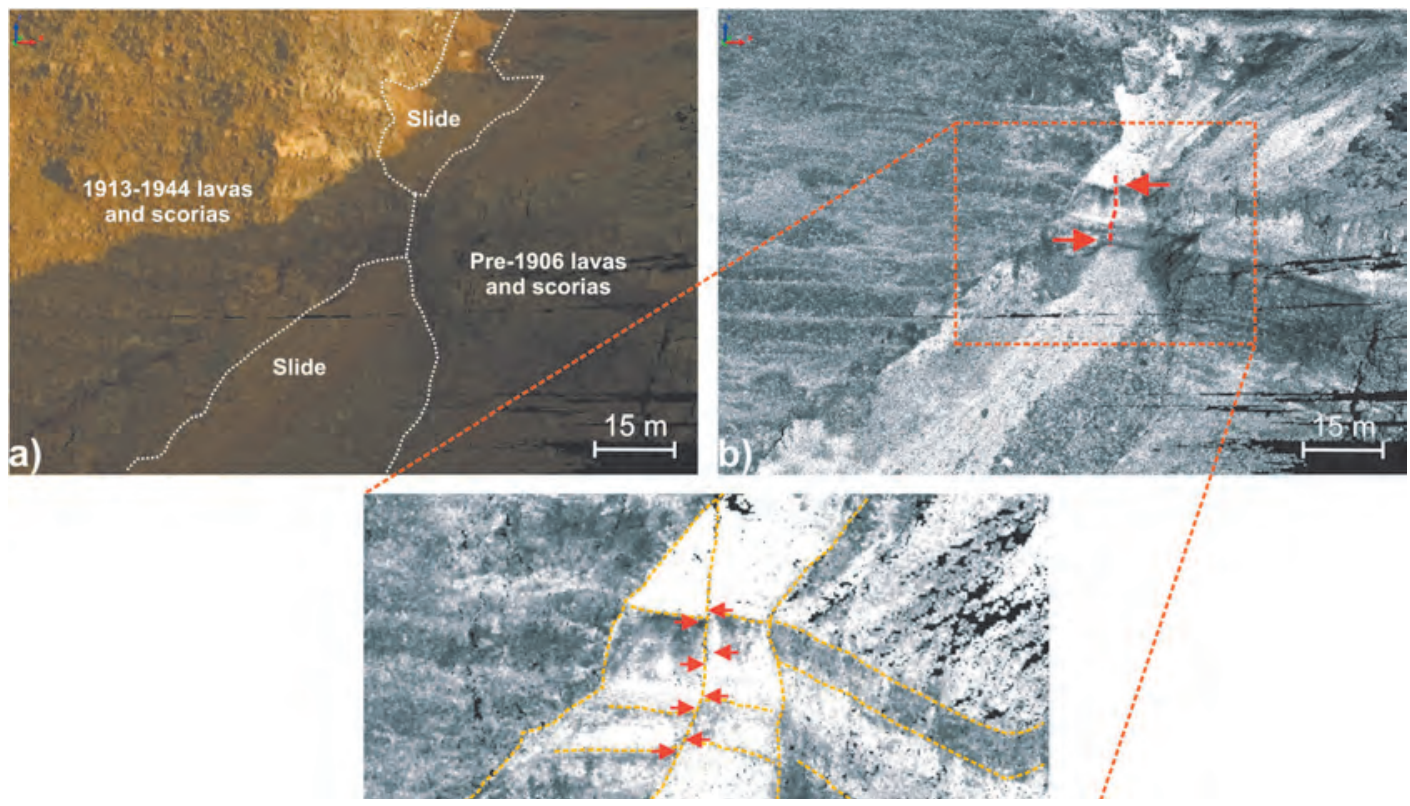

Fig. 3a,b. A-zone is a fracture zone in the SE part of the Vesuvius crater. The point cloud is colored using the RGB texture (a) and the intensity data (b). The zoomed area shows a vertical discontinuity of a high intensity horizontal layer, leading to a step of about $1.5 \mathrm{~m}$. In order to better recognize the fracture zone, the line-of-sight is far from the normal to the surface. The $15-\mathrm{m}$ scale is referred to the square-framed zone only.
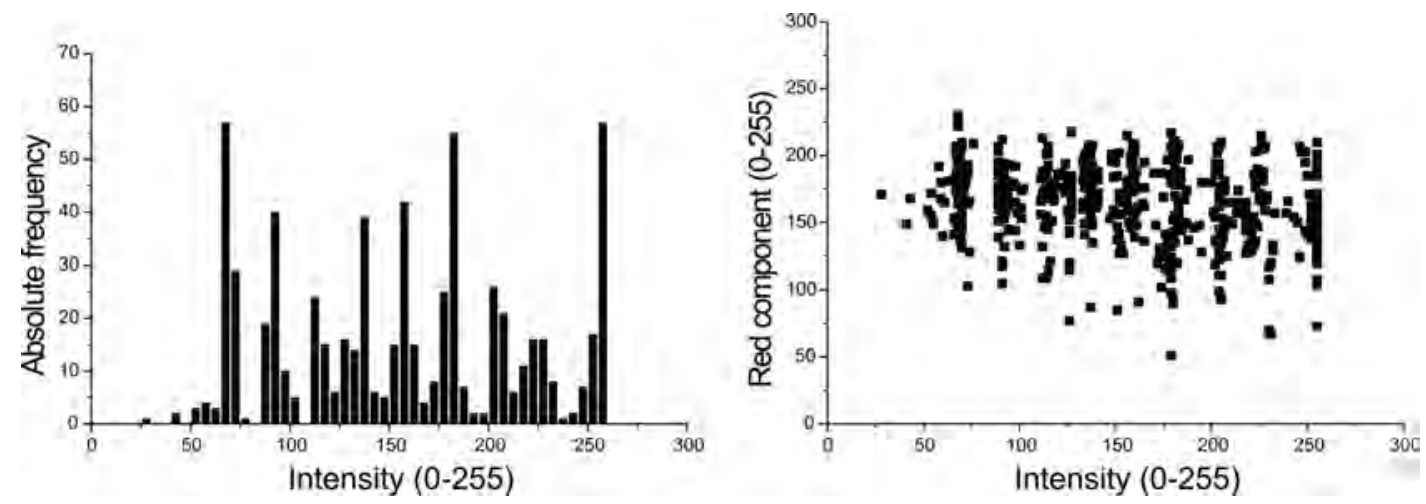

Fig. 4a,b. Statistical distribution of intensity data extracted from the vertical profile 3 (a), and plot of TLS-based intensity vs. red channel of the RGB image (b).

effect of the TLS detector, whose dynamics could be unable to capture the entire range of received signal intensity. Note that the quanti- zation effect, which is an instrumental artifact, can be also sharply observed in those conditions where the comb-peak pattern does not ap- 
pear neither in the vertical sections, nor in the entire surface. Moreover, the comb-peak effect and the quantization one differ by one order of magnitude. Therefore, the comb-peak effect seems to be not related to instrumental troubles. To exclude possible systematism, a further site area was selected and analyzed (C-zone, fig. 2).

\subsection{Landslide}

This study case (C-zone in fig. 2) is the central sector of a landslide affecting the northwestern inner wall of the crater. $\mathrm{C}$-zone was selected because its material (chaotic lava blocks and loose scorias) is the same as the B-zone, but it is not stratigraphically organized. C-zone represents a debris/fall slide. Figures $5 \mathrm{a}$ and $5 \mathrm{~b}$ show intensity data statistical distribution and correlations, respectively. The lack of a well defined point distribution (no comb-peak shape is observed in this case) and the detection of almost the same intensity peaks leads to the conclusion that the comb-peak shape, if it exists, is not an artifact but is related to material properties.

The distributions of the intensity peaks of vertical sections related to the volcanic wall and landslide are compared. Data are fitted by Gaussian functions over the peak intensity frequencies considering an a priori sigma of 10 in- tensity points. Figure 6 shows the results in terms of central values of Gaussian functions, with errors at $1-\sigma$ level. The landslide data show larger errors, whereas the volcanic wall data are clearly described by function with a very sharp shape. Nine peaks are selected for each data set, with similar values taking into account errors. The main discrepancies are highlighted in the figure.

A further test is performed using data belonging to a non-volcanic environment. See Appendix A, where results related to a coal quarry in South Africa are presented. These results underlie the relation of intensity and environment and partially reinforce the exclusion of instrumental effects.

\section{Discussion and data interpretation}

The analysis of the data from the B-zone of Vesuvius highlights the lack of correlation between intensity and colors. In particular, the correlation coefficient computed using all the points is about -0.06 value. Further computations, performed over some vertical surface boxes of the inner wall of the crater are shown in fig. 7. Data distribution confirms the occurrence of comb-peak-shaped trends, even if it can be easily recognized in the case of vertical profiles only, since the noise is lower and the
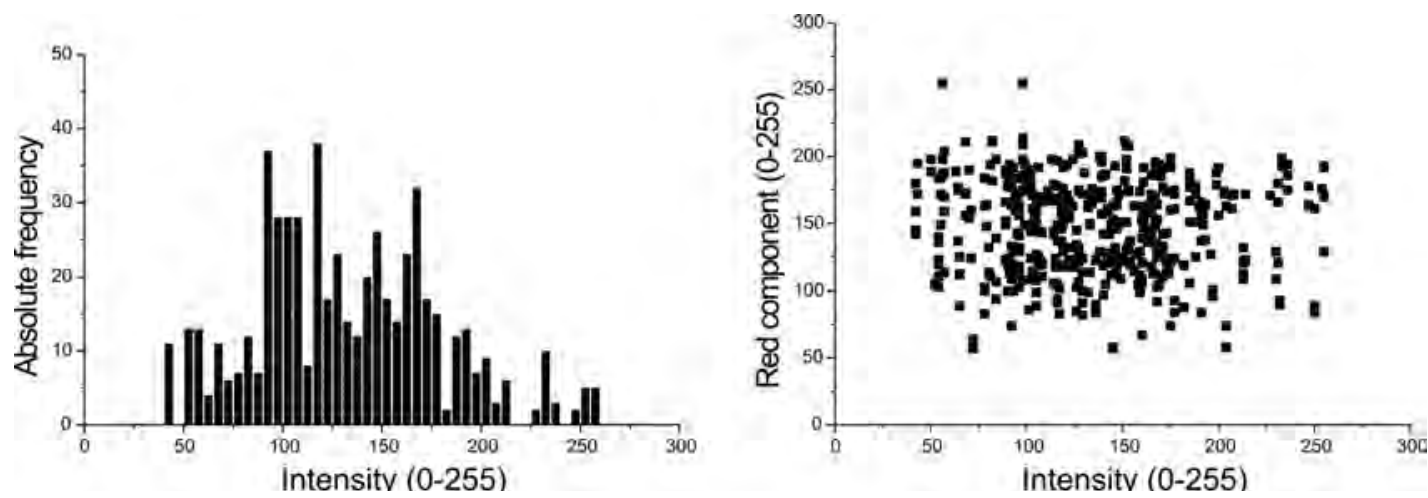

Fig. 5a,b. Statistical distribution of intensity data extracted from the vertical profile of the landslide (a), and plot of intensity vs. red channel (b). 


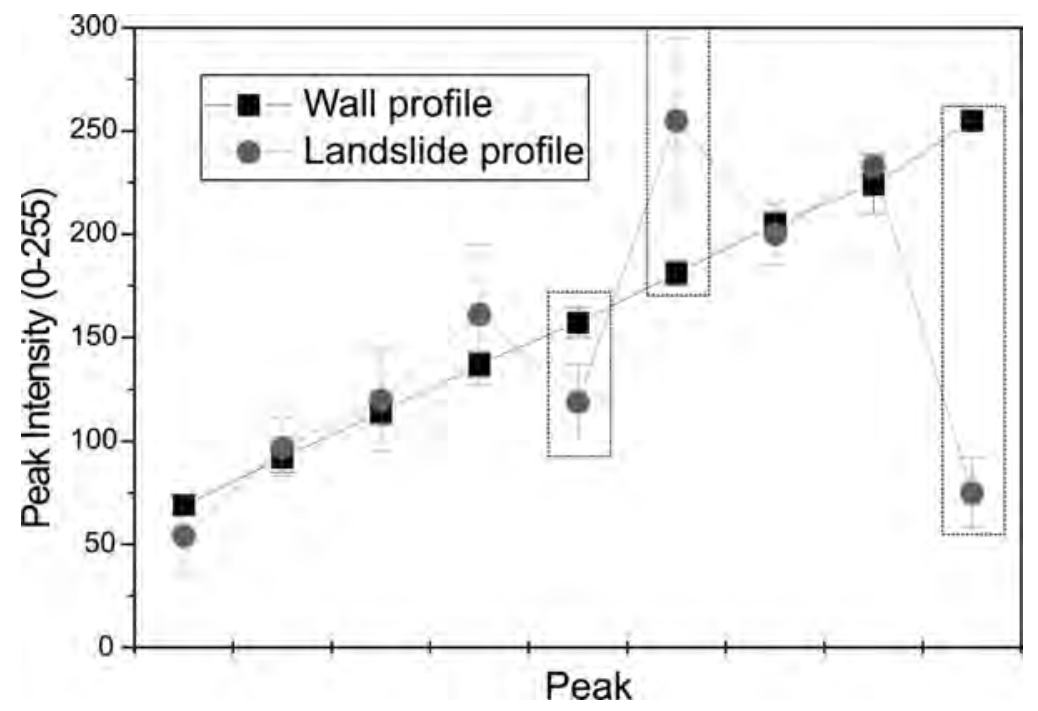

Fig. 6. Peaks distribution comparisons: red and black points are referred to landslide and volcanic wall respectively. Yellow boxes evidence the discrepancies.
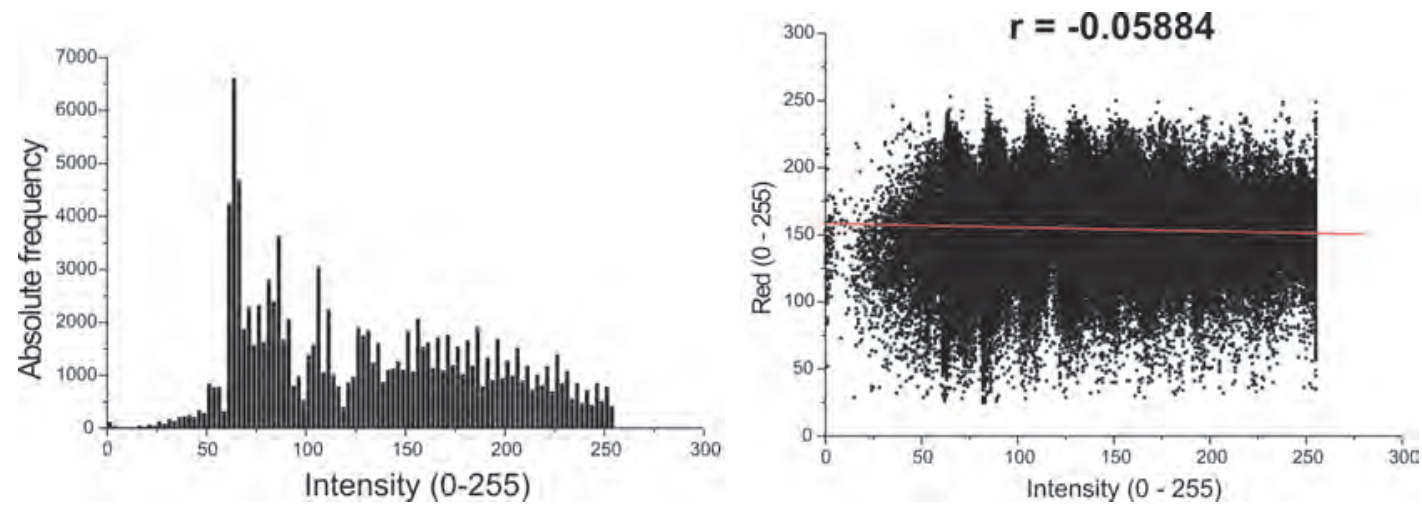

Fig. 7. Statistical distribution of the complete intensity data set extracted from the whole volcanic wall (a), and red channel and Intensity correlation (b).

number of data is reduced.

This consideration can be supported considering data from some vertical surface bands instead of the whole data set or some profiles only. In this way a large number of data can be considered but limited to almost the same vertical zones represented by previous profiles. Figure 8 shows results concerning $5 \mathrm{~m}$ width bands located at the left, at the center and at the right of the site-test volcanic wall. The main peaks are newly computed and evidenced in the statistical distribution histograms but now, the noise is higher, leading to fatter Gaussians in the peak finder analysis.

Also in this case the data are processed by recognition of significant peaks in the distribu- 

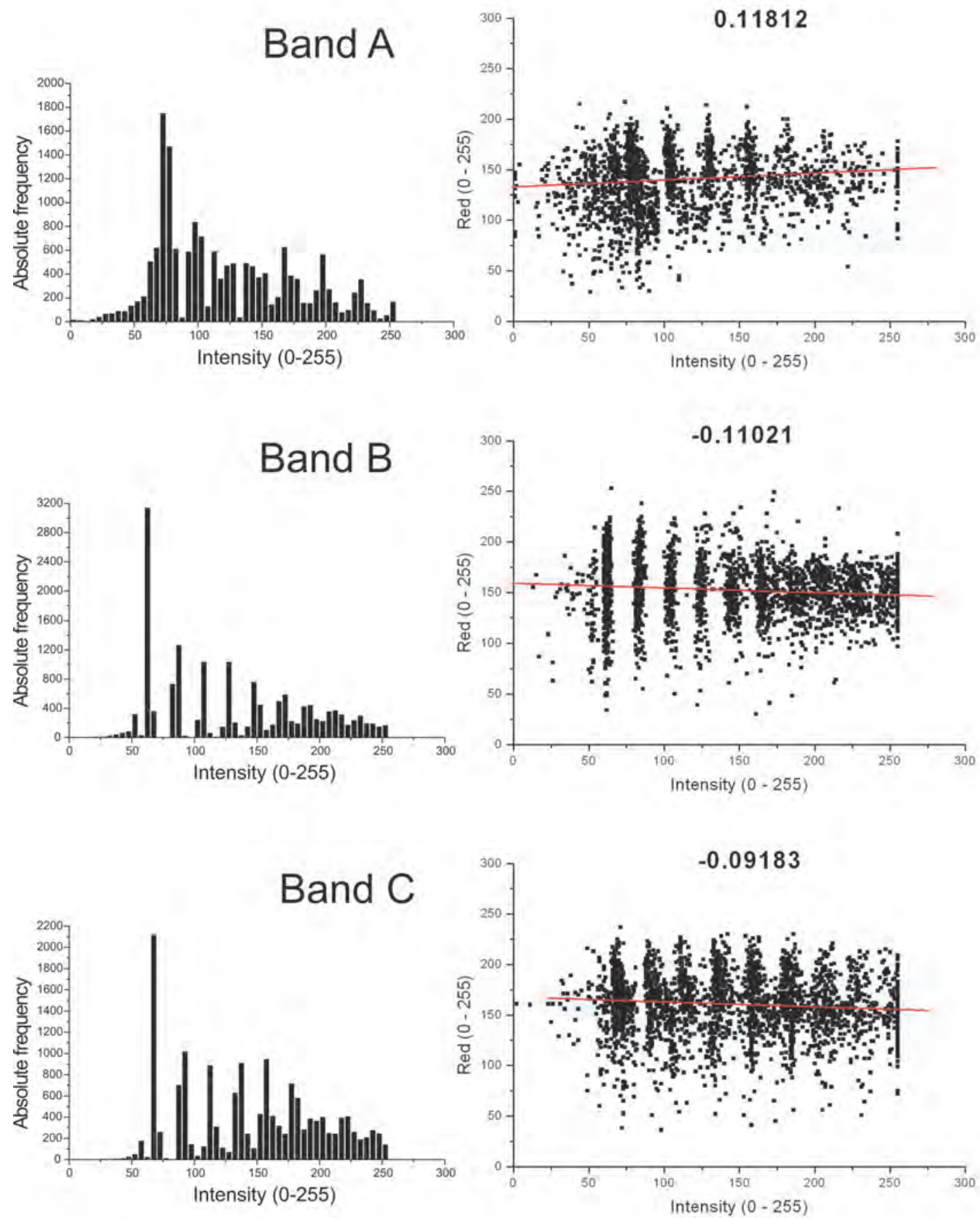

Fig. 8. Statistical distribution and data correlation of 3 data sub-sets. Also in these cases, despite larger noise, peaks are well visible. 


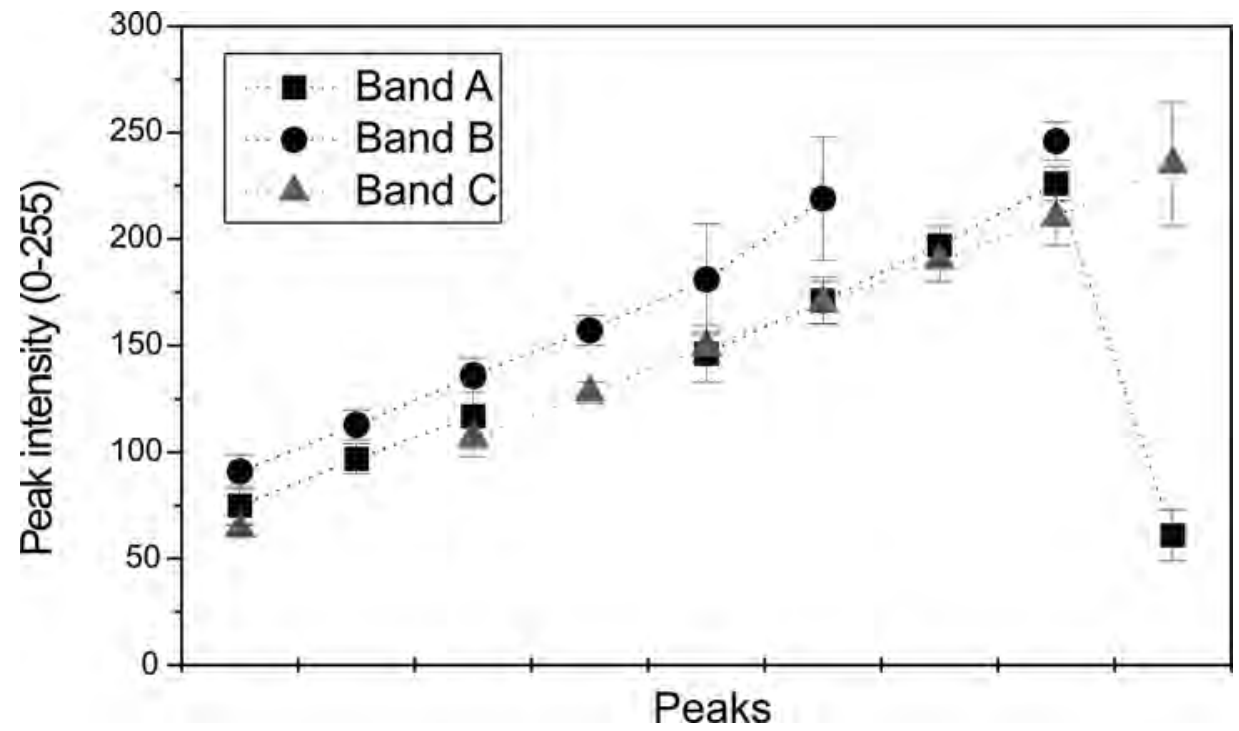

Fig. 9. Intensity peaks recognized considering three vertical bands $5 \mathrm{~m}$ width in the B-zone.

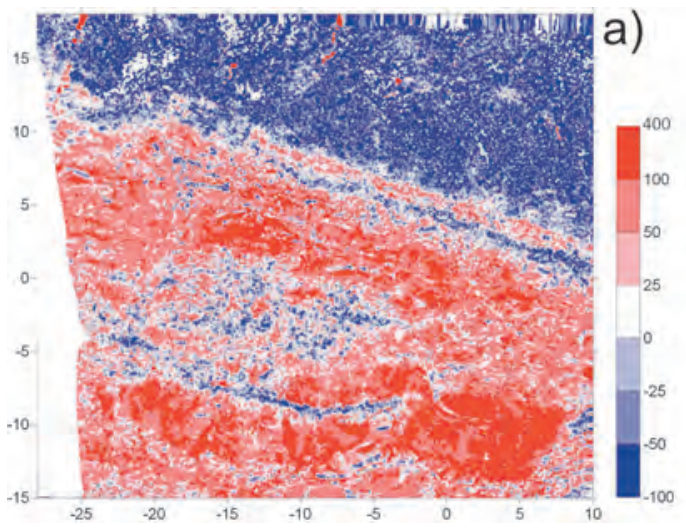

b)

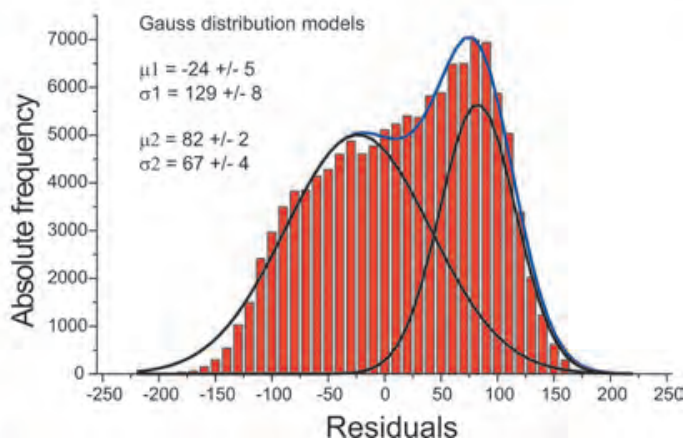

Fig. 10. Residuals map of B-zone and their statistical distribution. The residuals are computed as difference between intensity and R component of the RGB image (see also fig. 4). The distribution is not-normal, but can be considered as overlap of two normal distributions whose means and standard deviations are also reported.

tion. For each vertical band, mean and standard deviation of the Gaussian distribution are estimated and shown in fig. 9.

Another global surface study was carried out by means of estimation of residuals obtained from the difference of intensity and col- or data. The residuals are shown in figure 10 and point out different patterns. The B-zone residuals have a mean value of 18 , a standard deviation of 71 , and a non-normal distribution. The latter may result from the sum of two normal distributions peaked at 70 and -30 . A clear 

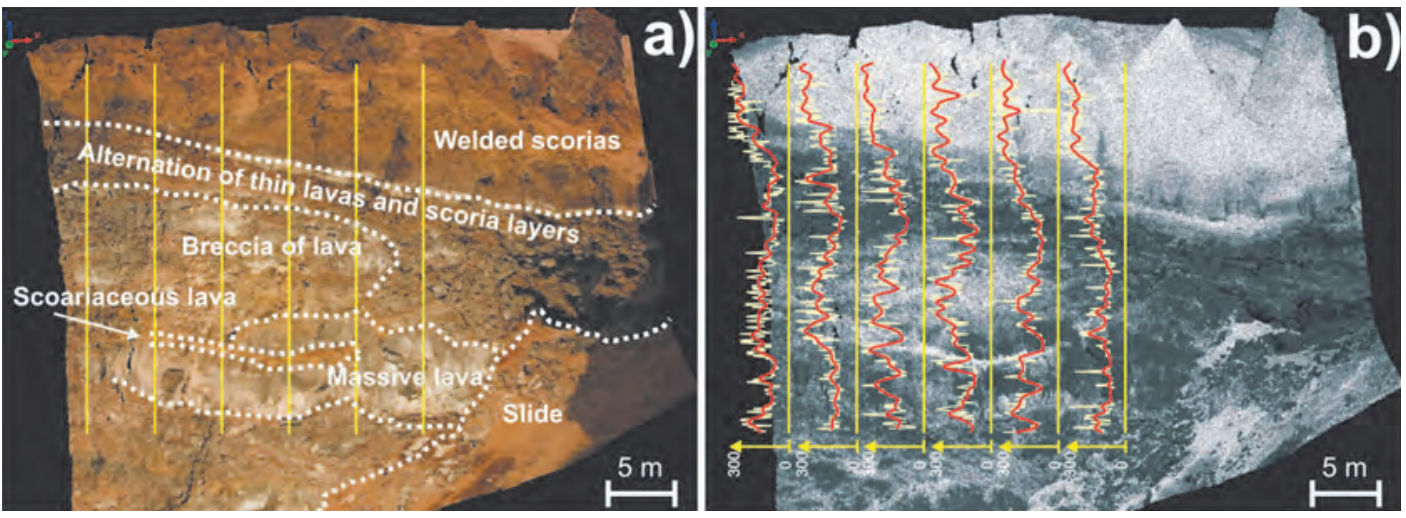

Fig. 11. Intensity of the six profiles selected in the B-zone (fig. 4). Data (in white) are smoothed (red line) using a FFT filter to better display slow variations and examine the intensity trend along the sections. A common scale of 0-300 is adopted.

boundary between the positive (red) and negative (blue) values of the residuals can be detected in the map.

The analyses presented above on the difference between intensity and color data are very simple and directly relate different datasets affected by major noise. For this reason, a principal components analysis (PCA) was also performed on data images by computing the most meaningful basis to re-express a noisy data set (Jolliffe, 2002; Shlens, 2005). This analysis transforms the input data so that the elements of the obtained vectors are uncorrelated. In addition, the number of vectors obtained may be reduced by retaining only those components that contribute more than a specified fraction of the total variance of the dataset. In this way, only the meaningful and no redundant information is retained, and the possibility of easy detection of particulars in the transformed images is associated to a significant reduction of the data storage requirements.

A standard PCA procedure of analysis was applied using Matlab functions (see Appendix B) to process a full data matrix composed by the RGB and the Intensity data sets. Results show the independence of the two data set, confirming the correctness of previous analysis. The same procedure was also applied to demonstrate that intensity information is inde- pendent from the stationing point of the instrument in this configuration of acquisition (Appendix C). In fact, the surface natural corrugation and the footprint have sizes that allow the laser beam to be diffused along each angles in the space, providing the same signal amplitude neglecting the geometry of acquisition.

Data extracted from A-zone clearly show a $1.5 \mathrm{~m}$ long vertical step (fig. 3). This step corresponds to the zone where a fracture is mapped by Ventura et al. (2005). The collected data indicate that this fracture is not an open crack, but a fault separating the products of the 1913-1944 activity from those of the pre-1906 activity. The age of the younger deposits affected by this fault and the $1.5 \mathrm{~m}$ slip evidenced from TLS data suggest an average slip rate of $2.4 \mathrm{~cm} / \mathrm{y}$. However, the slip rate along this fault cannot be constant over time, and could be related to a single or few post-1944 faulting episode(s). Intensity profiles from the zone B (fig. 4) highlight the transition from the uppermost welded scoria layer of the 1944 eruption, characterized by high intensity values, and the underlying lavas of the 1913-1944 period of activity, which show lower values. More in detail, the profiles also mark the boundaries between the massive and scoriaceous portions of the lavas (see profiles $2-5$ in fig. 11).

The rough boundary between the lavas and 
the upper welded scoria layer is also evidenced in the map of the residuals (fig. 10). However, the residuals do not clearly discriminate the scoriaceous portions of the lavas from the massive ones (see fig. 4 for a comparison). To better analyze the geological features of the Bzone, the cloud of points was divided in intensity bands at intervals [50, 80], [120, 150], $[150,200]$ and $[220,255]$ based on peaks distribution. These intervals were obtained computing the main Gaussian peaks of the six profiles, performing averages and creating intensity boxes to efficiently subdivide the data for material characteristics detection. Results of this analysis are shown in fig. 11. The image with intensity range [50- 80] highlights the massive portions of the 1913-1944 lavas depicted in fig. 4, whereas the images with intensities in the ranges $[150,200]$ and $[220,255]$ show the sco- riaceous levels. The image in the range [120, 150] marks the boundaries among the different lithological units. Therefore, we conclude that the integrated analysis of the residuals, intensity profiles and bands gives useful information on the lithology and stratigraphy of volcanic deposits.

Data from the C-zone clearly show the different layering of the lavas in the N-W and S-E inner walls of the Vesuvius crater. These slides are related to both gravity instability and earthquake shaking (Ventura et al., 1996). The point cloud shows the spatial extent of the debris/fall slide separating the two sectors of the Vesuvius crater, the source area, the summit scar, the slide wedge and basal fan (see fig. 6). Based on the grey intensity of the slide material, a more recent (shining pixels in fig. 13) slide deposit can be recognized from the older slides. The di-
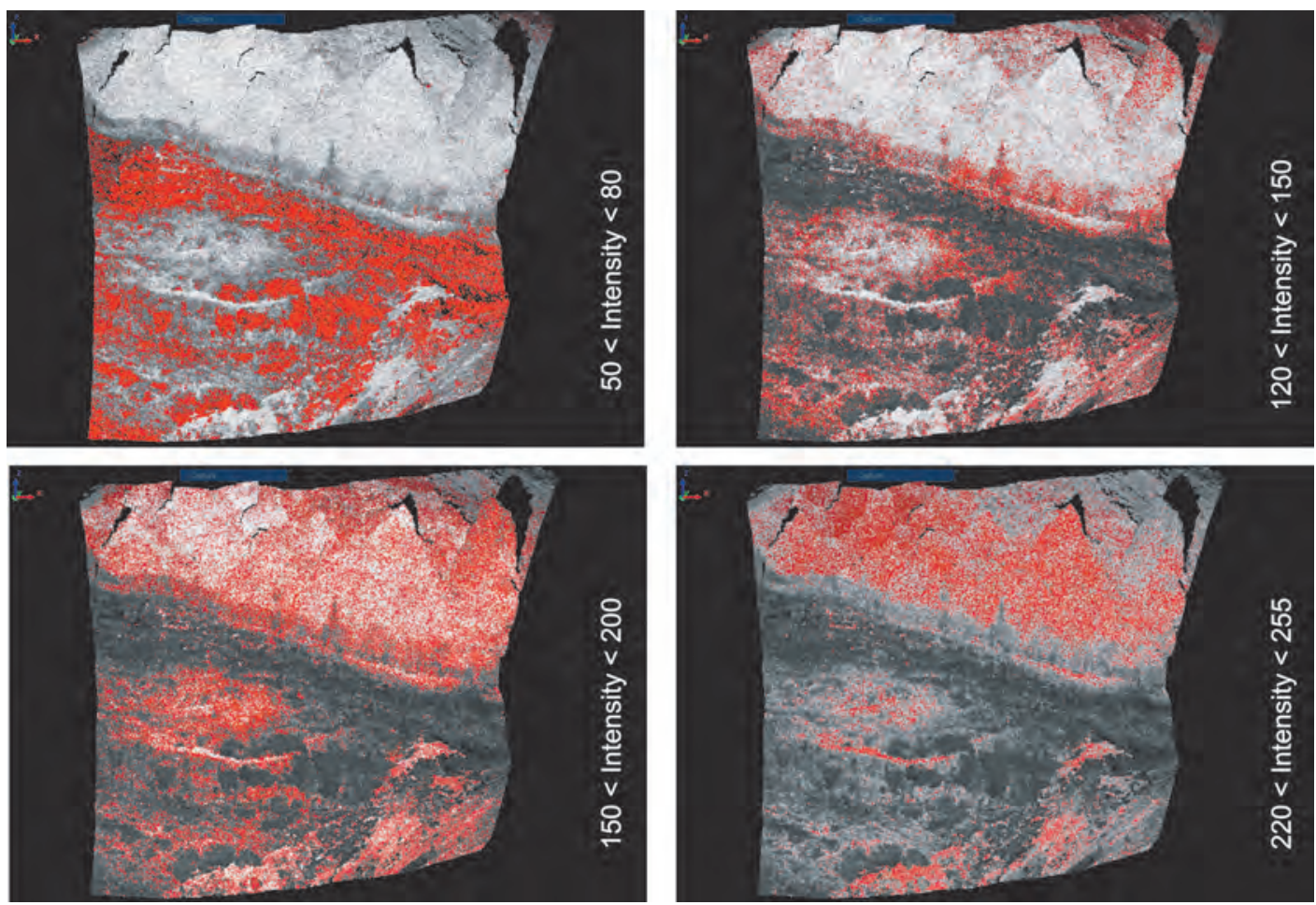

Fig. 12. Selection of the points of the B-zone on the basis of the intensity ranges. Four ranges are considered: [50- 80], [120-150], [150-200] and [220, 255]. 

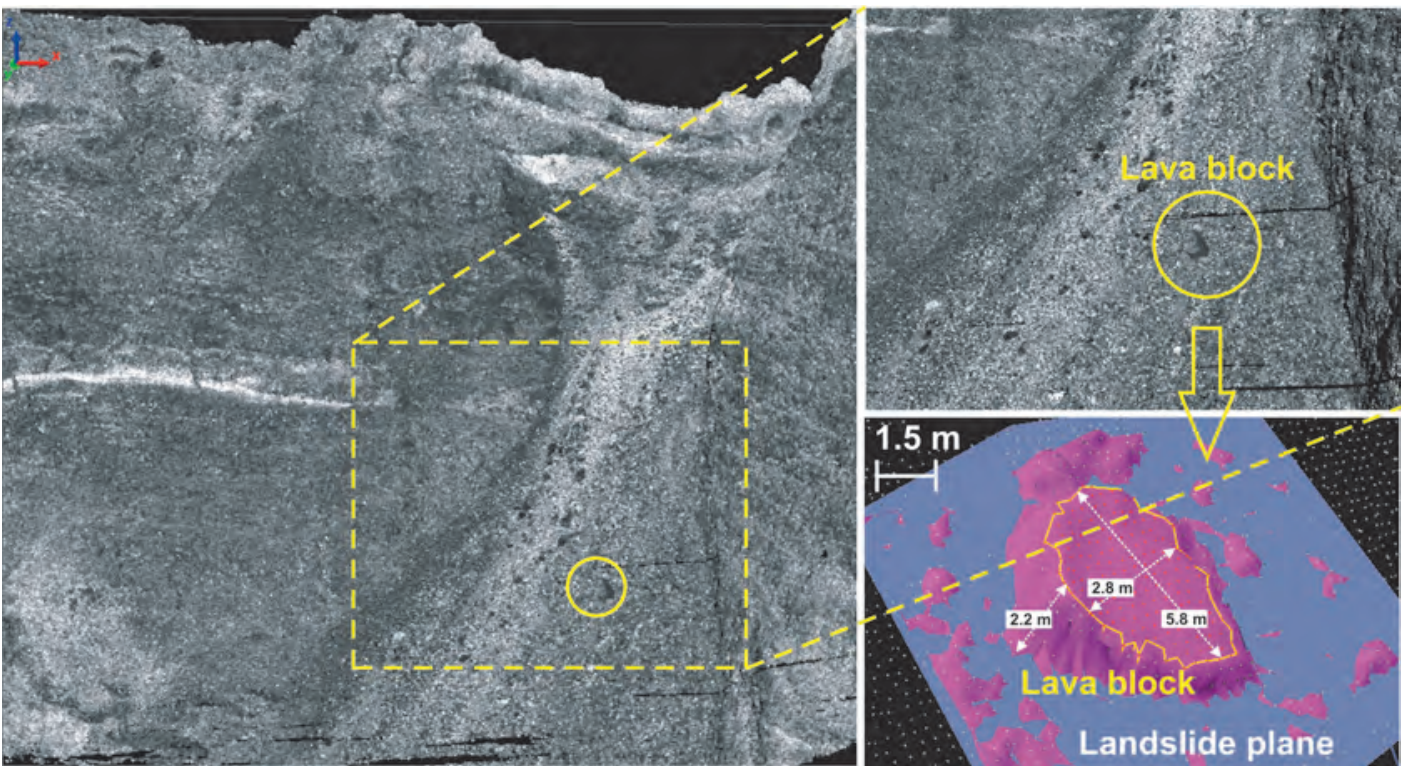

Fig. 13. Point cloud of the C-zone (landslide in volcanic environment, see fig. 6). The detail of a slide lava block and the size of the modeled surface are shown.

mensions of the slide material can be also determined using point cloud coordinates. As an example, the largest block of this slide measures $3.7 \mathrm{~m} \times 2.4 \mathrm{~m} \times 1.6 \mathrm{~m}$.

The integration of TSL data and the geological knowledge of the Vesuvius volcanic crater allow the recognition of some patterns: dark zones highlight lava blocks, data belonging to the second interval seems to be familiar with the contact area between different lithologies, and the higher values are probably due to pyroclastic materials of different size.

\section{Conclusions}

This study focuses on the analysis of radiometric data in volcanic environments and investigates the difference between color information content and TLS-based IR intensity. In particular, the RGB data from a calibrated camera can be independent or highly correlated to IR, depending on the geological environments, the intensity being strictly related to the physical/chemical properties of the material. Some selected sites of the Vesuvius crater were tested to investigate the relation between RGB and intensity, and to give preliminary results on the impact of such an approach on geological studies. Results show that intensity data are independent enough from RGB adding valuable information to image analysis, which can be considered a different channel, as shown by both a classical data treatment and PCA modern processing. The data analyzed here allow:

i) discrimination between different lava flow faces, block and welded scorias,

ii) identification of the layering of volcanic deposits, and

iii) recognition of slide material and sliderelated morphologies. In addition, quantitative estimates of the dimension of single clasts can be done. These applications and analyses represent a preliminary approach to validate a new method for data inspection based on integration between TLS data and photographic images.

The analytical approach reported here could be extended to other non-volcanic environ- 
ments, as suggested by the example of a coal quarry. Researches in this field are in progress and the next step includes (a) monitoring slide movements within the Vesuvius crater, and (b) planning a multidisciplinary experiment based on scanning of an artificial surface composed of several elements disposed to reproduce a synthetic, stratified, surface. Concerning the second point, a new experiment was performed in April 2008 showing that the intensity data is almost independent with respect to the incident angle if the observed surface irregularities are of the same order of the spot size. A complete description of the experiment and results is now submitted on Annals of Geophysics journal and partially satisfies the search for a new method to better analyze intensity data and their implication in geophysical/geological studies.

\section{Acknowledgments}

The authors thank Fabiana Loddo (INGV), Andrea Faccioli and Marco Bacciocchi (Codevintec Italiana s.r.l.) and Dario Conforti (Optech Inc.) for their kind support in the experimental phase.

Very special thanks to Massimiliano Cerrone and to all the staff of the «Ufficio Tecnico» (INGV-Rome) for their great contribute in the realization of an international seminar concerning Laser Scanner applications on 6-7 June 2007.

\section{Appendix A}

Results reported in chapter 4 about on intensity and color statistical distribution and their correlation show that data are widely independent, suggesting that important information can be added for a geological and geomorphological study of the area. In particular, the simple analysis of volcanic wall data set, shows a peak distribution (authors named «comb-peak shape»). Several considerations and data checks were done to exclude systematism, data alteration or instruments anomalies to verify the results reliability. Moreover, the same analysis performed on a landslide in a different part of the crater shows a completely different statistical distribution but with similar intensity values.

This Appendix reports the results of a further test performed in a non-volcanic environment (coal quarry at Middelburg, Moumalanga Province, South Africa) using the same instrument (Optech ILRIS 3D). With this additional test, the peculiarities of a volcanic area with respect to another geological environment can be highlighted. A single scan of a $25 \mathrm{~m}$ x $25 \mathrm{~m}$ vertical wall was acquired from a distance of $100 \mathrm{~m}$. After modeling and RGB data rendering, both intensity and color information are extracted along a profile (fig. A1). Results are quite different from those of the previous study cases, showing a clear correlation between the two kinds of data (fig. A2).
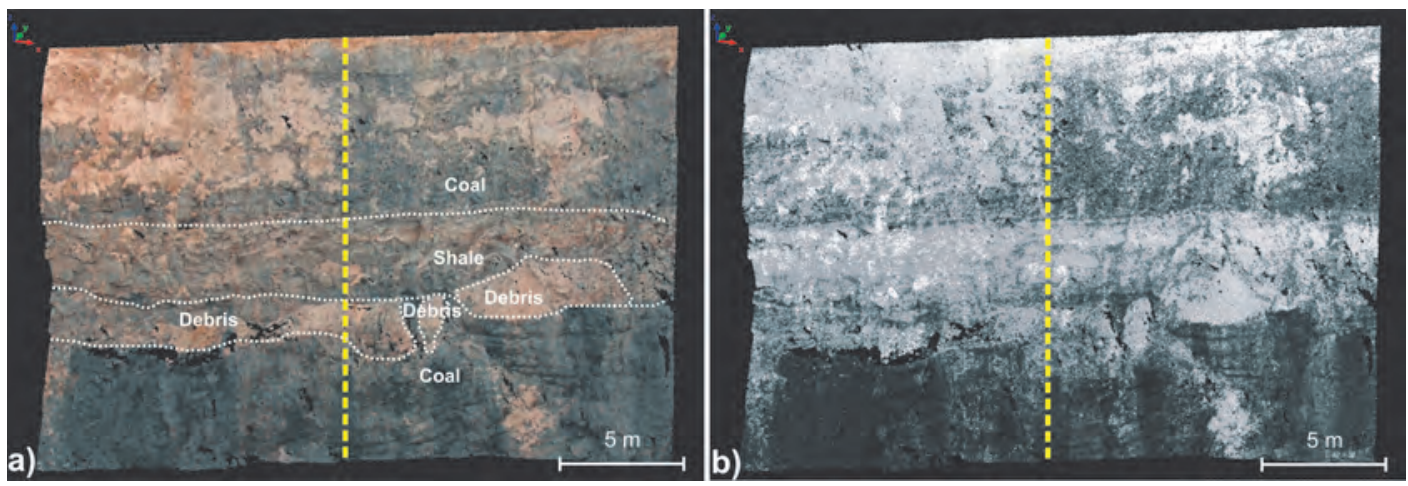

Fig. A1.Point cloud of a coal quarry (South Africa): RGB texture (a) and intensity image (a). The dashed yellow line is the selected profile. 


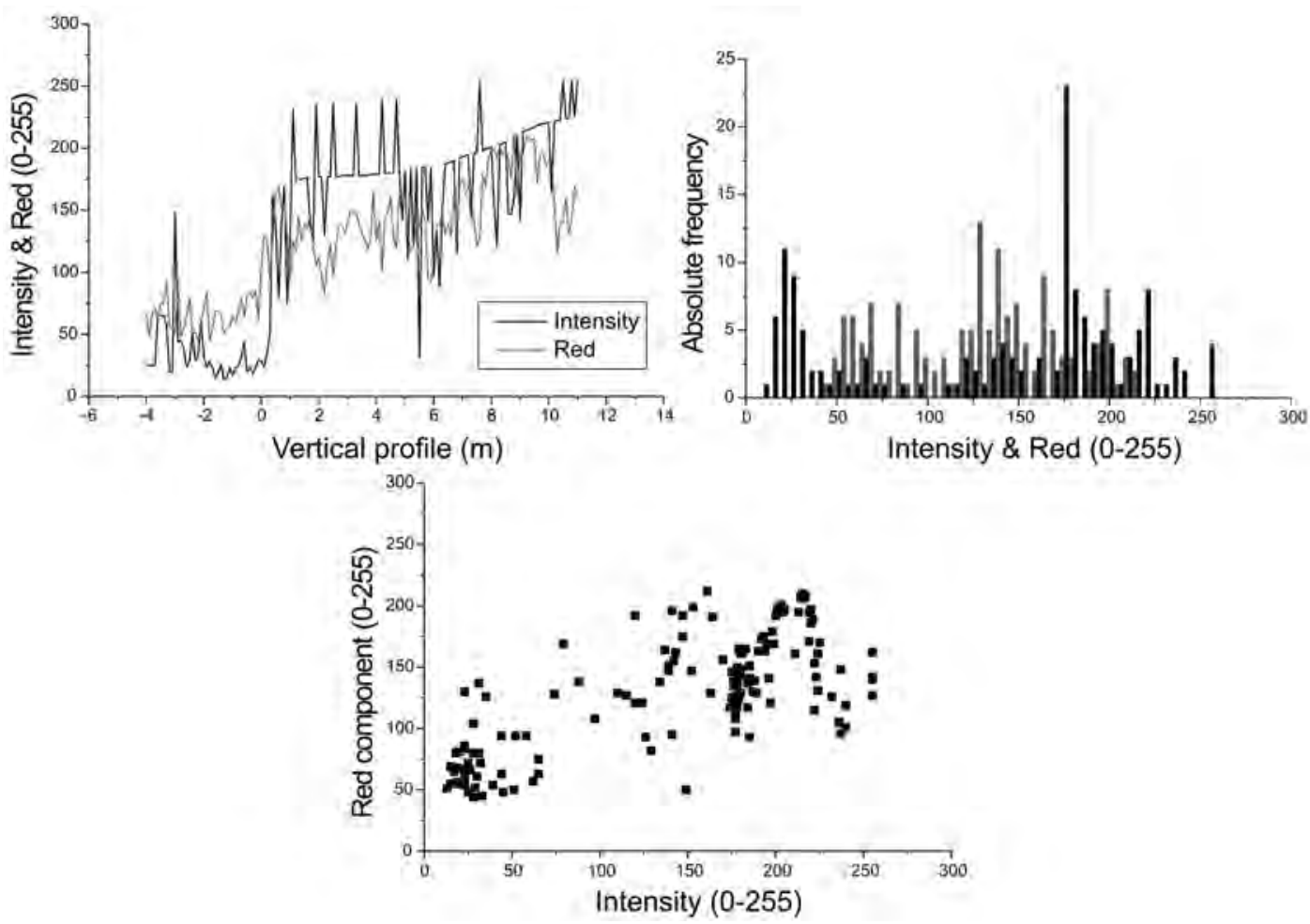

Fig. A2. Correlation between intensity and image levels along the coal quarry profile. Also this image shows great differences respect to the cases of volcanic zones.

In a first approximation, a linear trend can be recognized, even if the linear correlation coefficient is $\sim 0.6$. However, the high noise level, as well as the absence of correlation in volcanic environments, suggest that this relatively low value reflects, in any case, some data dependence. The statistical distribution analysis shows a lower number of intensity peaks with respect to that of the $\mathrm{B}$ and $\mathrm{C}$ volcanic sites. This can be related to the different analyzed surfaces and material properties. This analysis is not discussed here because it was performed only for comparison reasons to show that different geological environments give different responses.

\section{Appendix B}

Despite the evidence of a clear distribution, data belonging to the volcanic wall are characterized by high noise. Therefore, a more specific analysis was performed to validate the results reported in chapter 5, considering an approach based on principal component analysis (PCA). The PCA allows a reduction of redundant data and an easy recognition of details and hide features; in this case the method is used to highlight correlations between intensity and RGB data set.

The PCA application requires a preliminary transformation of all the data (the three RGB channels and the intensity data) to have zero mean and variance 1 . The results of PCA are still four datasets, but they are uncorrelated and ordered in a way that their participation to the data variance highly decrease from the first to the fourth. So, the less significant datasets can be excluded considering a limit-variance, e.g. 0.02. In the present work, Matlab ${ }^{\text {TM }}$ package was used to perform the PCA via the singular value decomposition (Golub and Reinsch, 1970). 


\section{a) 0.05}

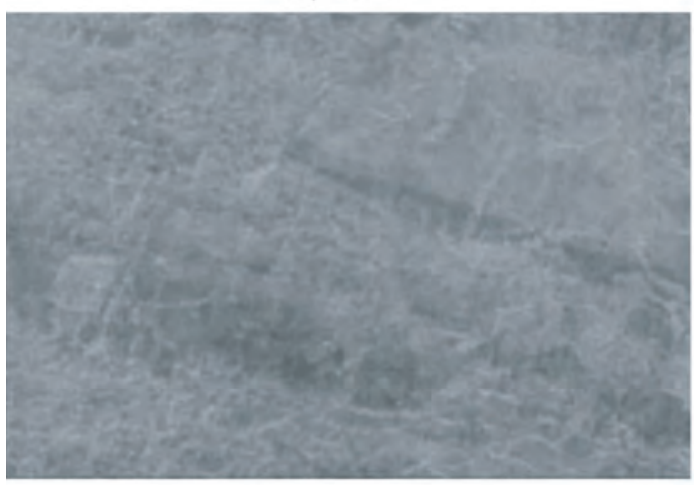

c) 0.06

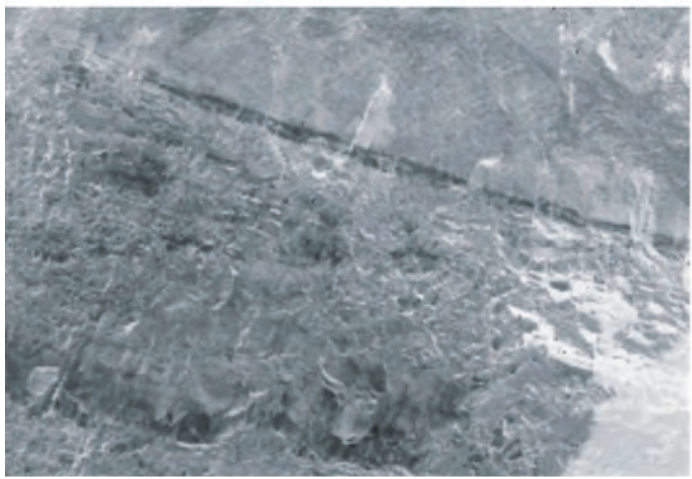

b) 0.05

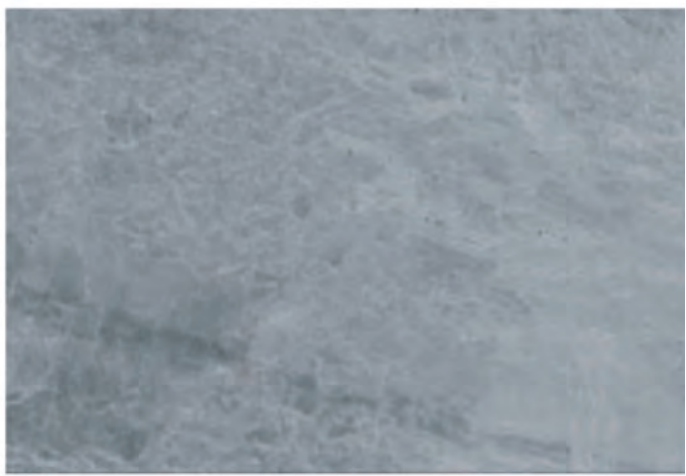

RGB image

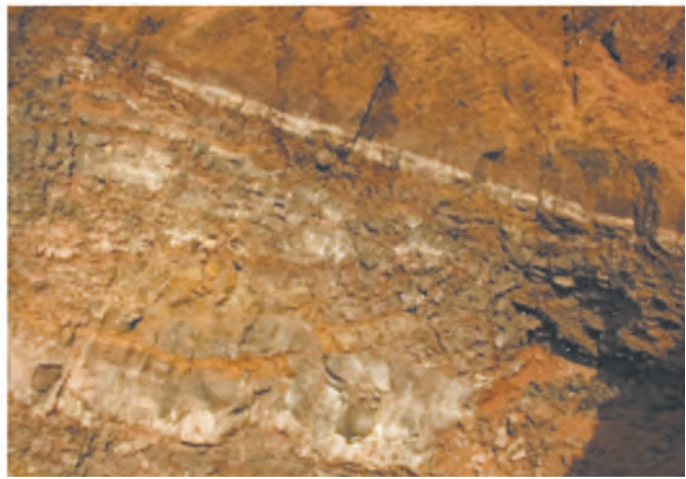

Fig. B1. Principal component analysis of the RGB image of a selected part in the B-zone: the principal components $(a, b)$, a virtual image obtained choosing 0.06 variance (c), the initial RGB image (d).

Input vectors were multiplied by a matrix whose rows consist of the eigenvectors of the input covariance matrix, producing transformed vectors. Clearly, all the transformed vectors, in particular the ones characterized by the higher variances, can contain information extracted from all the original input vectors.

For a given portion of the investigated surface, the RGB image and the intensity data can be considered. After registration on a common reference frame, the original data were interpolated to provide two images having exactly the same size. The RGB image is represented by three $N$-by- $M$ matrices and the intensity data by one $N$ by- $M$ matrix. Each of these matrices is transformed into a row vectors having $N M$ length. If an RGB and an intensity image are considered, a 4-by- $N M$ matrix is therefore built. Thanks to PCA, the real color information, the relations between colors and intensity, as well as the implications of using the red component alone can be investigated. In particular, the following analyses were performed: i) PCA of the three components of the RGB image (three input vectors), ii) PCA of the R component and of intensity data (two input vectors), iii) PCA of the RGB and intensity data (four input vectors), and iv) PCA of the intensity data provided by TLS acquisition of a same area from two different viewpoints (two components). In all the cases, several variance-limits were considered.

In the first case the RGB image was considered, without intensity data. Only a vector is obtained from the PCA if a 0.06 limit variance was considered, and this fact is expected since the previous analyses have highlighted that the three color components are strongly correlated. The results are shown in fig. B1. 
a) 0.05

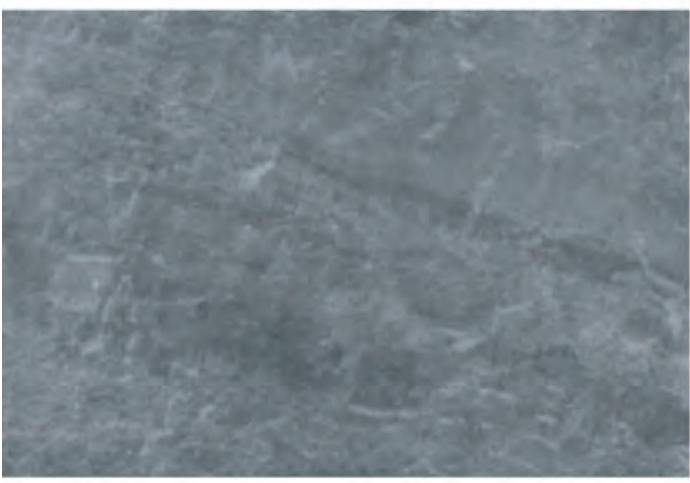

c) RGB

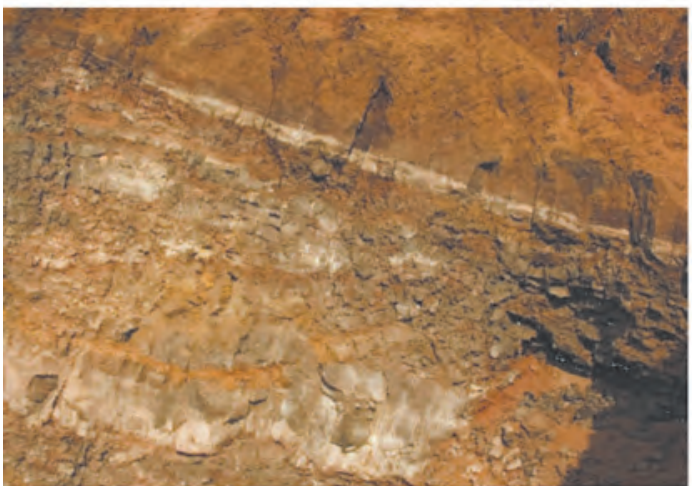

b) 0.05

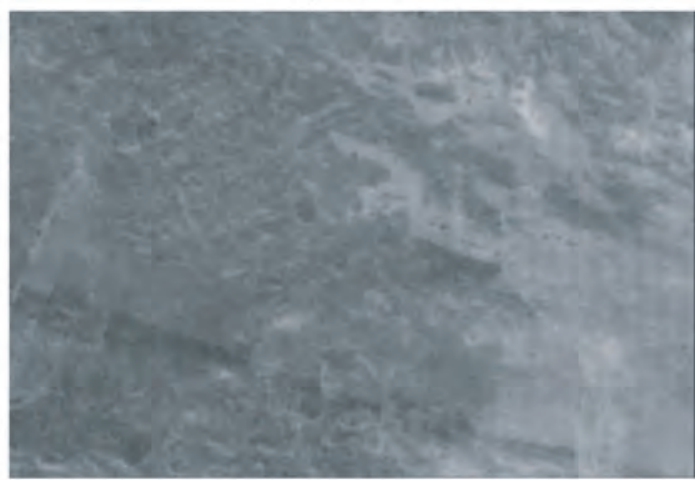

Intensity

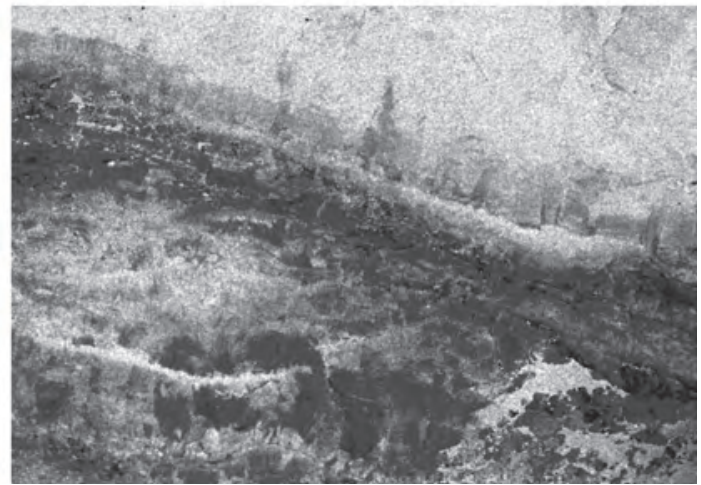

Fig. B2. Principal component analysis of R channel of the RGB image and TLS-based intensity: original R channel (a); intensity (b), principal component having the largest variance (c,d). Note that on this PC, which is the only output component if the 0.3 limit-variance is considered, contains information related to both the vectors, shows features that can be easily detected.

The results obtained in the second and third case are very similar, as expected. For this reason, only the results of the second analysis ( $\mathrm{R}$ component and intensity) are reported here. If a 0.05 limit-variance is considered, two matrices are obtained (fig. B2a). They cannot be combined because the synthesis of the two outputs would be very confusing. On the other hand, the principal component with largest variance ( 0.3 is the chosen limit-variance) contains all the information contained in both color and intensity image. All the intensity and red-component features are clearly visible, like the shadow in the right bottom of the image.

\section{Appendix C}

The PCA can be also used to check for intensity data reliability when the same area is acquired from two or more viewpoints, i.e. different distances and different geometrical conditions are considered. The angle of incidence affects the resulting intensity (Reshetyuk, 2006), but also the spot size and the surveyed area have to be considered. The surveyed volcanic surfaces are very corrugated with respect to the $5-10 \mathrm{~cm}$ illuminated element by the laser pulses. Therefore, no significant variations of the intensity data are expected. Figure $\mathrm{C} 1$ shows the 


\section{a) 0.05}

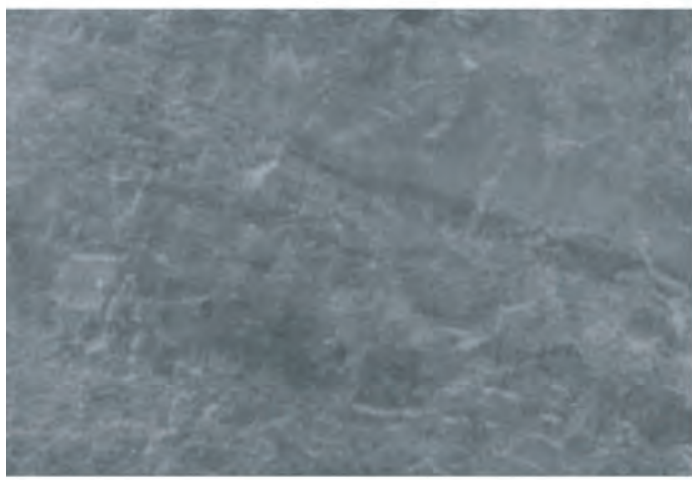

c) RGB

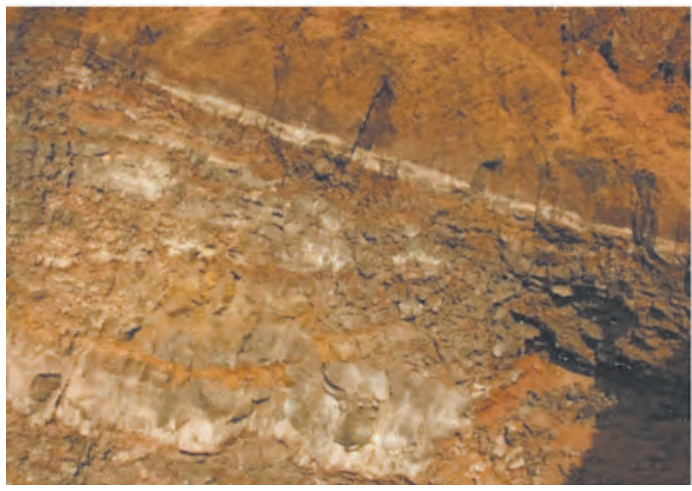

b) 0.05

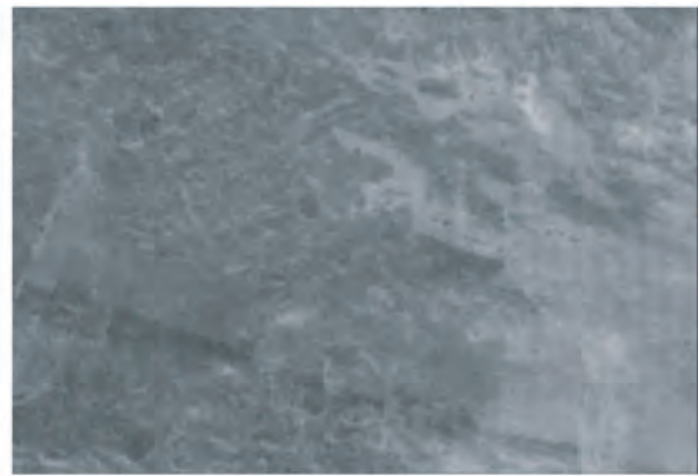

Intensity

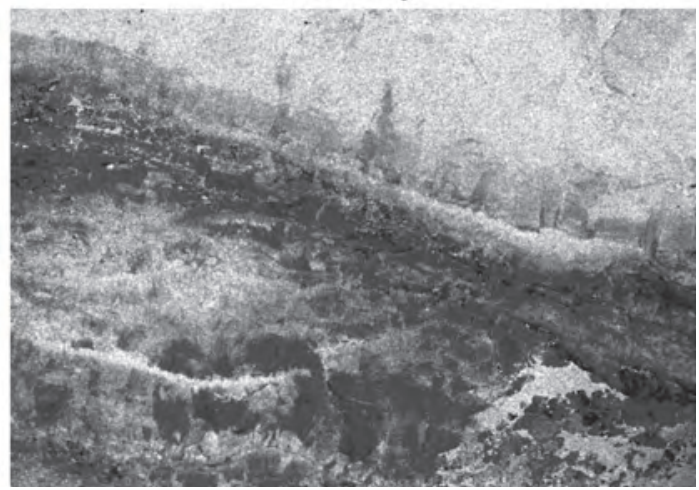

Fig. C1.PCA of the intensities in a case where two viewpoints are considered, with different distances and orientations: two scans with intensity data (a,b), transformed dataset, i.e. virtual image (c). All the features that can be separately detected in the two single scans can be easily found in the principal component.

results of PCA of the intensity patterns related to a same portion of the B-zone acquired from different viewpoints. The resulting image shows all the features related to both the input images.

In digital image processing, the PCA can be performed in another way. Instead of the application to a set of vectors representing several registered images, this technique can be applied to one image only. Boundaries and regions in a single image can be therefore described. This approach is not considered here, but will be applied in further analyses for automatic feature recognition purposes (in the present work the features were detected manually).

\section{REFERENCES}

Andronico, D., R. Cioni and R. Sulpizio (1996): General stratigraphy of the past 19,000 yrs at Somma-Vesuvius, in M. Rosi (Ed), Vesuvius Decade Volcano Workshop Handbook, (CNR, Napoli), pp. 1-21.

Arnò, V., C. Principe, M. Rosi, R. SAntacroce, A. Sbrana and M.F. SHERIDAN (1987): Eruptive history, in R. SANTACROCE (Editor), Somma-Vesuvius, Quaderni de «La Ricerca Scientifica», (CNR, Roma) 114, pp. 53-103.

BALTSAVIAS, E.P. (1999): Airborne laser scanning: basic relations and formulas, ISPRS Journal of Photogrammetry \& Remote Sensing, 54 (2-3), 199-214.

BESL, P.J. and N.D. MCKAY (1992): A method for registra- 
tion of 3D shapes, IEEE Transactions on Pattern Analysis and Machine Intelligence, 14 (2), 239-256.

Bianco, F., M. Castellano, G. Milano, G. Ventura and G. VILARDO (1998): The Somma-Vesuvius stress field induced by regional tectonics: evidences from seismological and mesostructural data, Journal of Volcanololgy and Geothermal Research, 82 (1-4), 199-218.

BoeHLer, W., M. Bordas Vicent and A. Marbs (2003): Investigating laser scanner accuracy, Proceedings of XIX CIPA symposium, edited by O. ALTAN (Antalya, Turkey, 30 Sept. - 4 Oct. 2003. Available online at: http://cipa.icomos.org/filead min/papers/antalya/189.pdf) (Accessed: 16.05.2007)

Chen, Y., G. Medioni (1992): Object modelling by registration of multiple range images, Image and Vision computing, 10 (3), 145-155.

Hunter G., H. Pinkerton, R. Airey and S. CAlvari (2003): The application of a long-range laser scanner for monitoring volcanic activity on Mount Etna, Journal of Volcanology and Geothermal Research, 123 (12), 203-210.

Golub, G.H. and C. ReINSCH (1970): Singular Value Decomposition and Least Squares Solutions, Numerische Mathematik, 14 (5) , 403-420.

Jolliffe, I.T. (2002): Principal Component Analysis, (Springer Verlag, Berlin Heidelberg (Ed), New York), 487, pp. $180-213$

Kurazume, R., K. Nishino, Z. Zhang and K. IKeuchi (2002): Simultaneous 2D images and 3D geometric model registration for texture mapping utilizing reflectance attribute, in Proceedings of the 5th Asian Conference on Computer Vision (ACCV), I, 99-106.

Lichti, D.D. and S. JAMTSHO (2006): Angular resolution of terrestrial laser scanners, The Photogrammetric Record, 21 (114), 141-160.

Lu, J., A.S. Georghiades, H. Rushmeier, J. Dorsey and C. $\mathrm{Xu}$ (2005): Synthesis of Material Drying History: Phenomenon Modeling, Transferring and Rendering, in Proceedings of Eurographics Workshop on Natural Phenomena, edited by E. GALIN and P. Poulin, (Eurographics Association, Aire-La-Ville, CH), pp. 7-16.

MazZarini, F., M. PAReschi, M. Favalli, I. Isola, S. TaRQUINI and E. BosCHI (2007): Lava flow identification and aging by means of lidar intensity: Mount Etna case, Journal of Geophysical Research, 112 (B2): doi:10.1029/2005JB004166.

Pesci, A., F. Loddo and D. Conforti (2007a): The first terrestrial laser scanner application over Vesuvius (Napoli, Italy): the high resolution model of volcano crater, International Journal of Remote Sensing, 28 (1), 203-219.

Pesci, A., M. Fabris, D. Conforti, F. Loddo, P. Baldi and M. ANZIDEI (2007b): Integration of ground-based laser scanner and aerial digital photogrammetry for topographic modeling of Vesuvio volcano, Journal of Volcanology and Geothermal Research, 162 (3-4), 123-138.

Pesci, A., G. Teza, G. Ventura, M. Bacciocchi, D. ConFORTI, M. FABris, M. ANZIDEI and P. BALDI (2007c): Terrestrial laser scanner for volcano monitoring, (Available online at: http://www.codevintec.it/seminari/optech07/Volcano_Monitoring.pdf). (accessed 31 October 2007).

PoB, (2008): Laser scanner hardware and software survey. (Available online at: http://laser.jadaproductions.net/), (accessed 25 May 2008).

Remondino, F. (2003): From point cloud to surface: the modelling and visualization problem, in Proceedings of ISPRS International Workshop on Visualization and Animation of Reality-based 3D Models, edited by A. Gruen, Sh. Murai, J. Niederoest and F. Remondino (24-28 February 2003, Tarasp-Vulpera, Switzerland, working group V/6 (CD-ROM).

RESHETYUK, T. (2006): Investigation of the influence of surface reflectance on the measurements with the terrestrial laser scanner Leica HDS 3000. Zeitschrift für Geodäsie, Geoinformation und Landmanagement 2006 (2), 96-103.

Roncella, R., G. Forlani and F. Rremondino (2005): Photogrammetry for geological applications: automatic retrieval of discontinuity in rock slopes, in Proceedings of Videometrics, VIII, edited by J.-A. BERALDIN, S.F. El-HaKim, A. Gruen and J.S. Walton (San Jose, CA, USA, SPIE-IS\&T Electronic Imaging 5665. SPIE, Bellington, WA), pp. 17-27.

SAntaCroce, R. (1987): Somma-Vesuvius, Quaderni de «La Ricerca Scientifica», 114. Consiglio Nazionale delle Ricerche, Roma, pp. 220.

SHLENS, J. (2005): A Tutorial on Principal Component Analysis. (Available online at: http://www.cs.cmu.edu/ elaw/papers/pca.pdf) (Accessed: 18 May 2007).

Teza, G., A. Galgaro, N. Zaltron and R. Genevois (2007): Terrestrial laser scanner to detect landslide displacement fields: a new approach, International Journal of Remote Sensing, 28 (16), 3425-3446.

Ventura, G., G. Vilardo, G., Bronzino, G., Gabriele, R. NAPPI and C. TERRANOvA (2005): Geomorphological map of the Somma-Vesuvius volcanic complex (Italy), Journal of Maps, 1, 30-37.

Ventura, G., G. Vilardo and U. TAmmaro (1996). Earthquake-induced slides at Monte Somma Caldera (JulyAugust 1995, Somma-Vesuvius Volcanic Complex), Acta Vulcanologica, 8 (1), 115-118.

(received May 6, 2008; accepted May 29, 2008) 